\section{Introduction and Methods}

Accumulation of studies have shown that barrier dysfunction in the skin is closely related with UVB-induced skin inflammation. In our previous study, we found that a natural product from peanut, named ethanol extract of peanut sprouts (EPS), was found to have a potent antioxidant activity by showing suppressive activities of the induced expression of COX-2 and nerve growth factor expression in the compound 48/80-treated HaCaT cells, an in vitro mode for skin inflammation. Also, the anti-inflammatory activity of EPS was also confirmed in the animal model of contact inflammation of oxazolone-induced contact dermatitis of mice in vivo. With these backgrounds, we studied whether UVB-irradiation induced barrier dysfunction could be protected by EPS treatment via its antioxidant activity in NHEKs. As control experiments, NHEKs were also treated $\mathrm{N}$-acetyl cysteine (NAC), a well-known antioxidant, to prove the efficacy of EPS in all of following experiments.

\section{EPS}

EPS stock solution was measured to contain trans-resveratrol at $176.75 \pm 3.63 \mu \mathrm{g} / \mathrm{mL}$

(Positive antioxidant control: NAC)

Cell viability

- MTT assay of EPS in normal human epidermal keratinocytes (NHEKs)

Measurement of intracellular ROS levels

- UVB-induced intracellular ROS was detected with a confocal microscopy using a DCF-DA

ROS-scavenging activity in NHEKs

- Tested biomarkers for inflammation cytokines : Interleukin-6 (IL-6), IL-8

- Tested biomarkers for MAPK pathway: pERK

- Tested biomarkers for barrier dysfunction: Keratin 1, Filaggrin (FIL), Involucrin

ROS-mediated inflammation via phosphoAKT (pAKT)/ hypoxia-inducible factor- $\alpha$ (HIF-1a) pathway

- Tested inflammatory mediators for Akt-HIF-1 $\alpha$ pathway: pAKT, HIF-1 $\alpha$

\section{Results}

\section{Determination of optimal experimental conditions}

In our search to find out the optimal experimental conditions, MTT assay demonstrated that $<1.6 \mathrm{mg} / \mathrm{ml}$ of EPS and $<4 \mathrm{~mJ} / \mathrm{cm}^{2}$ of UVB were safe without cytotoxicity to perform our experiments. Therefore, the following experiments were performed by treating NHEKs with $1 \mathrm{mg} / \mathrm{ml}$ EPS and $4 \mathrm{~mJ} / \mathrm{cm}^{2}$ of UVB (Figure 1 ).

\section{EPS suppressed ROS production in UVB-induced NHEKs}

To unravel the cytoprotective mechanism of EPS and NAC, we tested whether they had a potential to inhibit the UVB-induced ROS production in NHEKs. In DCF-DA staining, UVB produced DCF-DA-positive ROS from NHEKs, which was blocked by EPS and NAC treatment, respectively (Figure 2 ).

\section{EPS downregulates expression levels of inflammatory biomarkers in UVB-irradiated} NHEKS

UVB-induced upregulation of biomarkers for inflammation cytokines, such as IL-6, IL-8, COX-2 and ERK1/2, could be suppressed by treatments with EPS and NAC, indicating that ROS plays a crucial role to produce inflammatory cytokines in NHEKs (Figure 3A-C). Keratin 1 , involucrin and filaggrin were also down-regulated by UVB irradiation, which were reversed by EPS and NAC treatments (Figure 3D).

\section{EPS suppressed inflammatory mediators of pAKT and HIF-1a in UVB-irradiated} NHEKS

In RT-PCR, UVB-irradiation induced the up-regulation of pAKT and HIF-1a in NHEKs, which were suppressed by treatments with EPS and NAC (Figure 4). The results suggest us that ROS plays a crucial role in inflammatory process via pAKT/HIF-1a pathway in keratinocytes

5. EPS suppressed the NF-kB-mediated inflammation in UVB-irradiated NHEKs

From our in vitro results to demonstrate the anti-inflammatory activities of EPS, we examined the regulatory effects of EPS by assessing $I_{\kappa B} \alpha$ expression levels in NHEKs. In real-time PCR and Western blot analyses, UVB induced the downregulation of $I_{\kappa} B \alpha$, an inhibitor of NF-KB, in NHEKs, which were reversed by EPS or NAC treatments. The results indicate that EPS and NAC have an anti-inflammatory activity by inhibiting NF- $\kappa B$ transcription in UVB-irradiated NHEKs (Figure 5A, B).

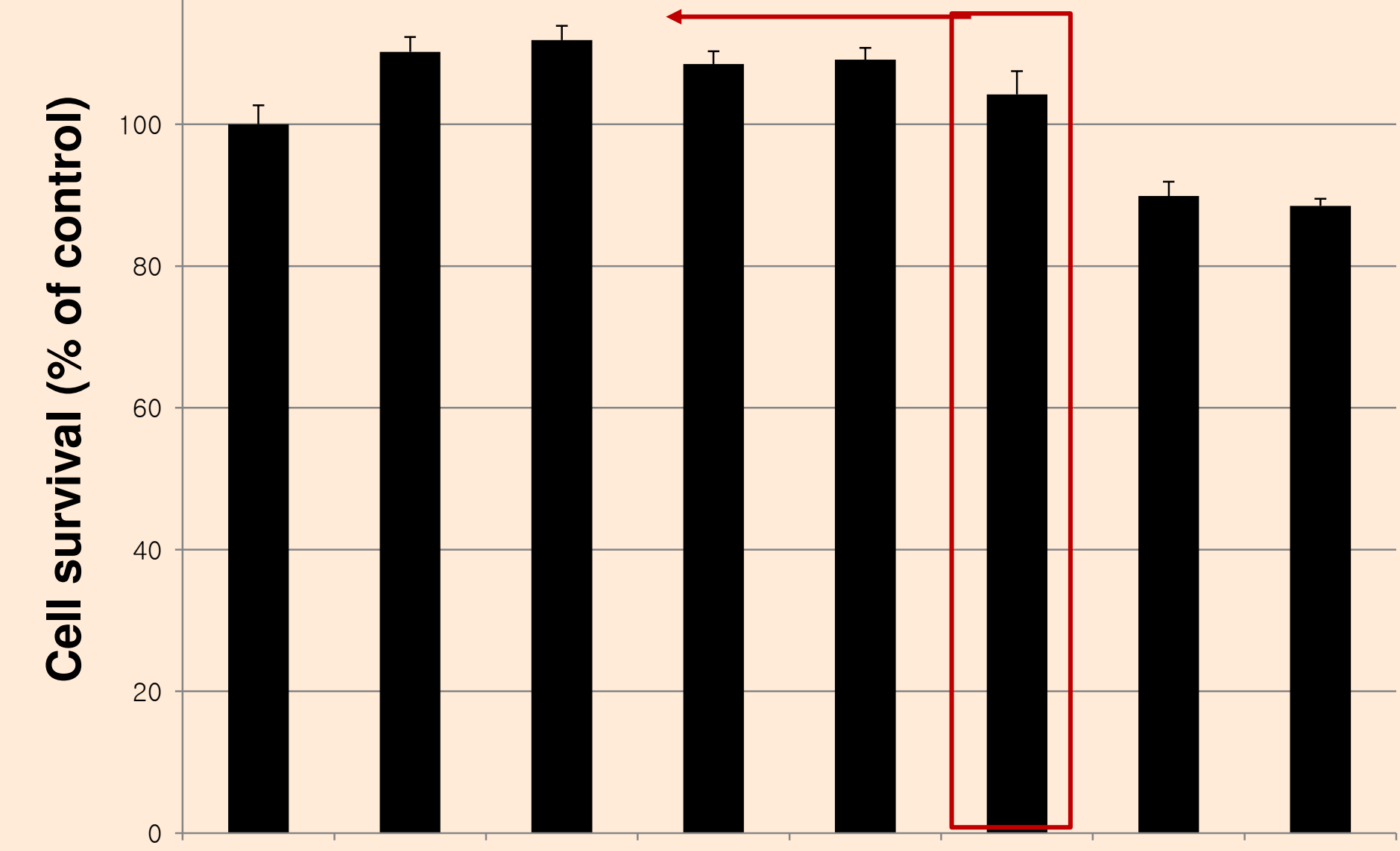

EPS Conc. $(\mathrm{mg} / \mathrm{ml})$

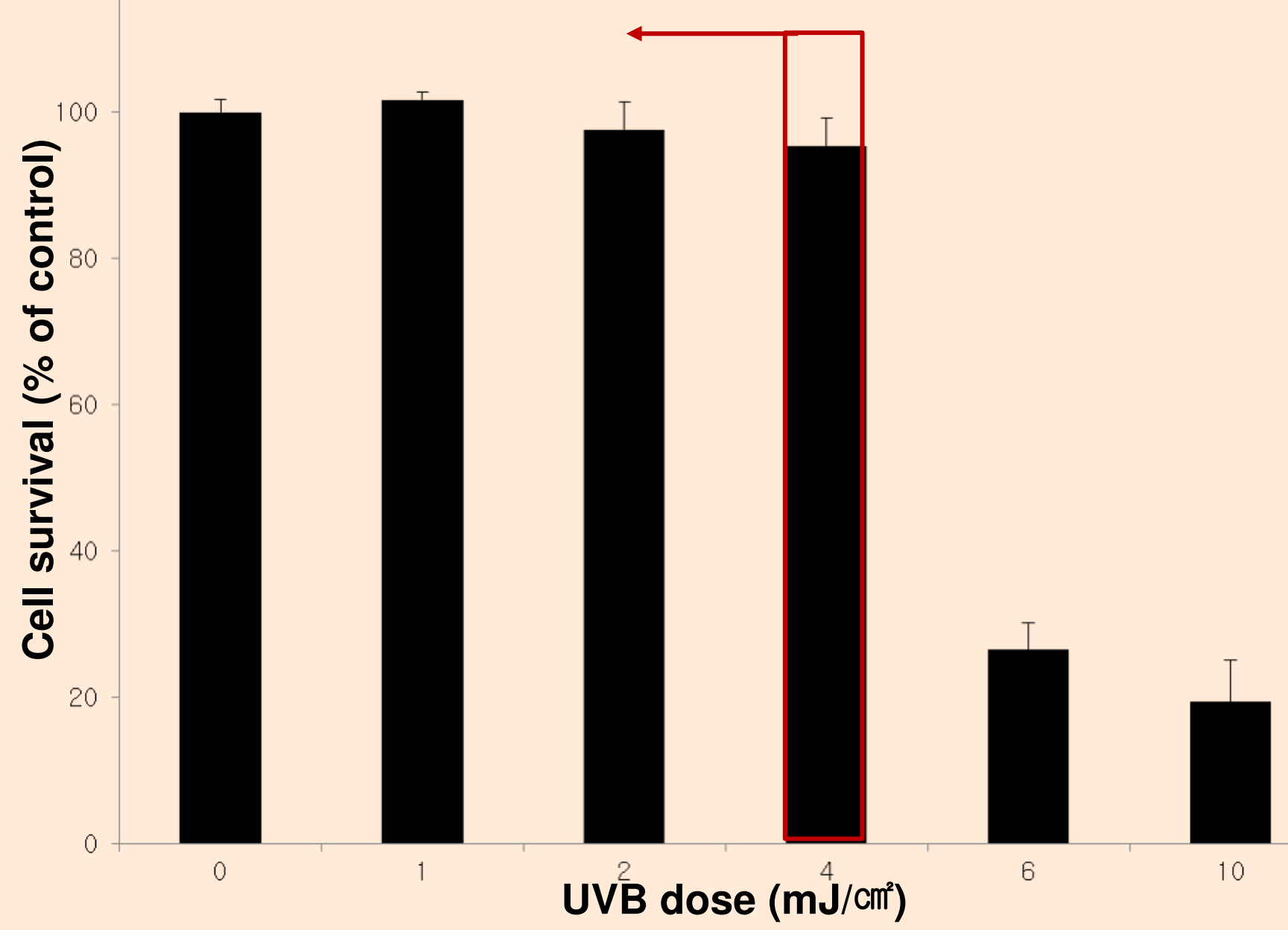

UVB dose
Figure 1. Set-up of non-cytotoxic conditions of EPS, UVB-irradiated NHEKs

MTT assay to test cytotoxicity was performed after NHEKs were treated with the EPS and UVB irradiation for $24 \mathrm{~h}$ to decide optimal concentration of EPS and UVB doses for our experiments.

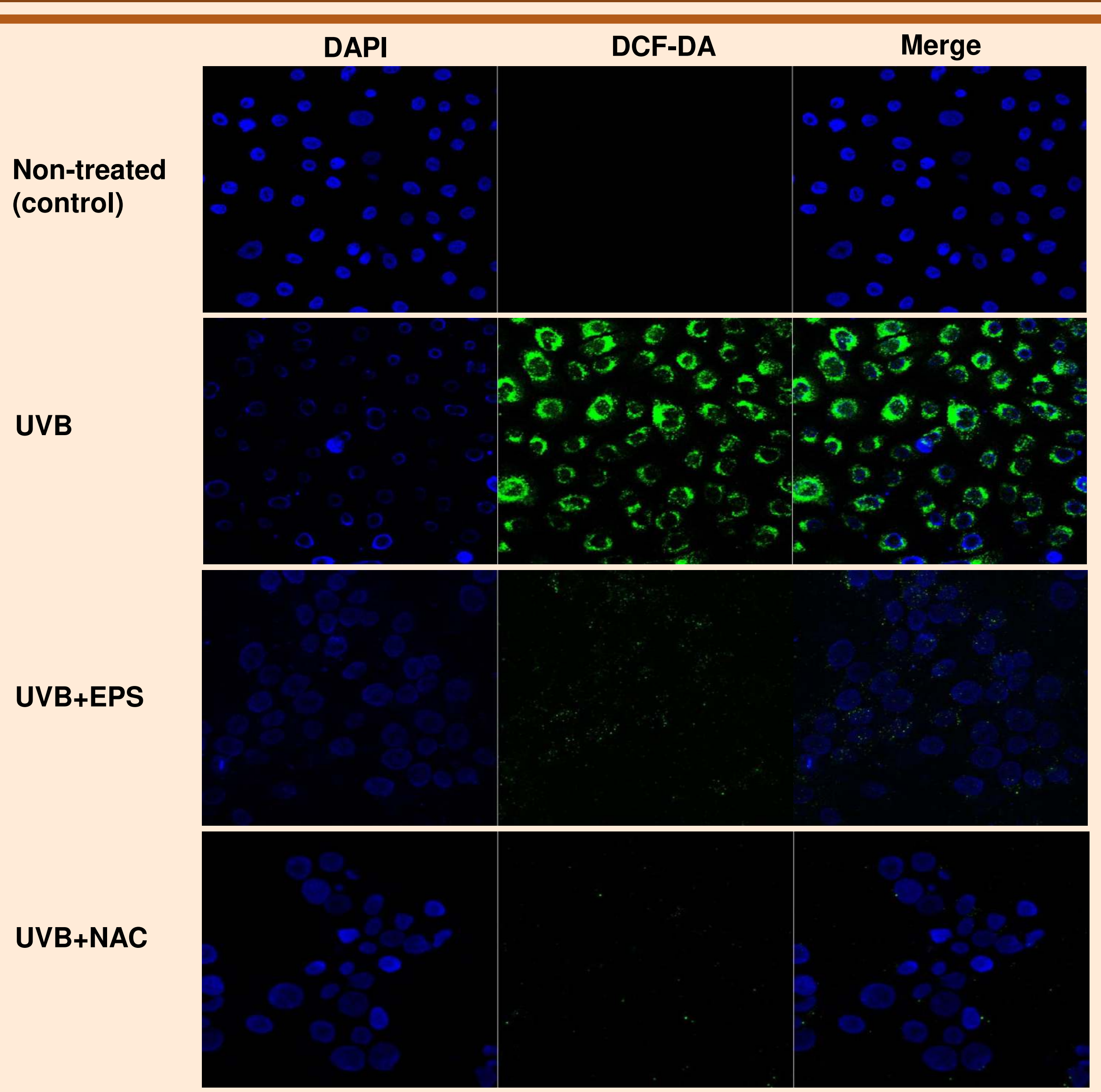

Figure 2. Effect of EPS on ROS production in UVB-irradiated NHEKs After NHEKs were irradiated with UVB, cells were labeled with DCF-DA dye to detect intracellular ROS by a confocal microscope. Control NHEK without UVB irradiation, UVB-irradiated NHEKs, EPS-treated, and NAC-treated UVBirradiated NHEK.

A
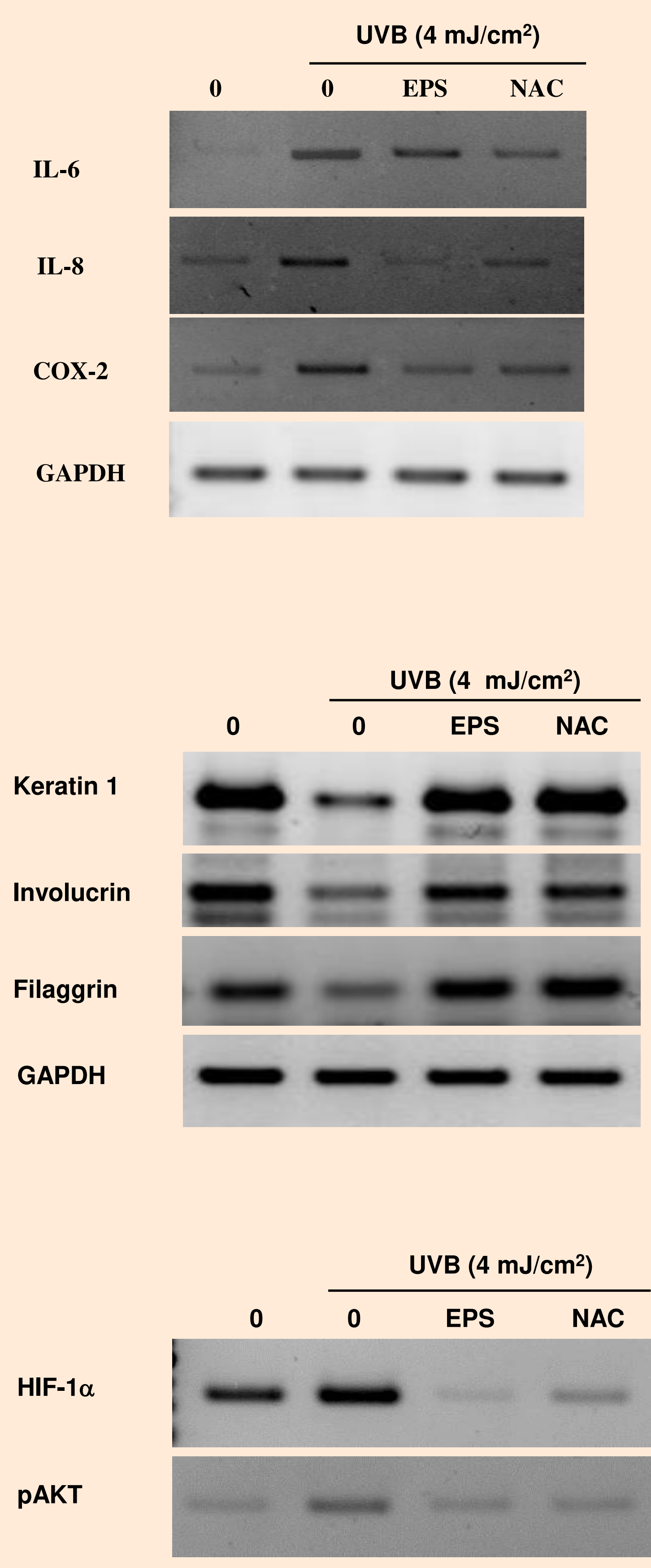

GAPDH

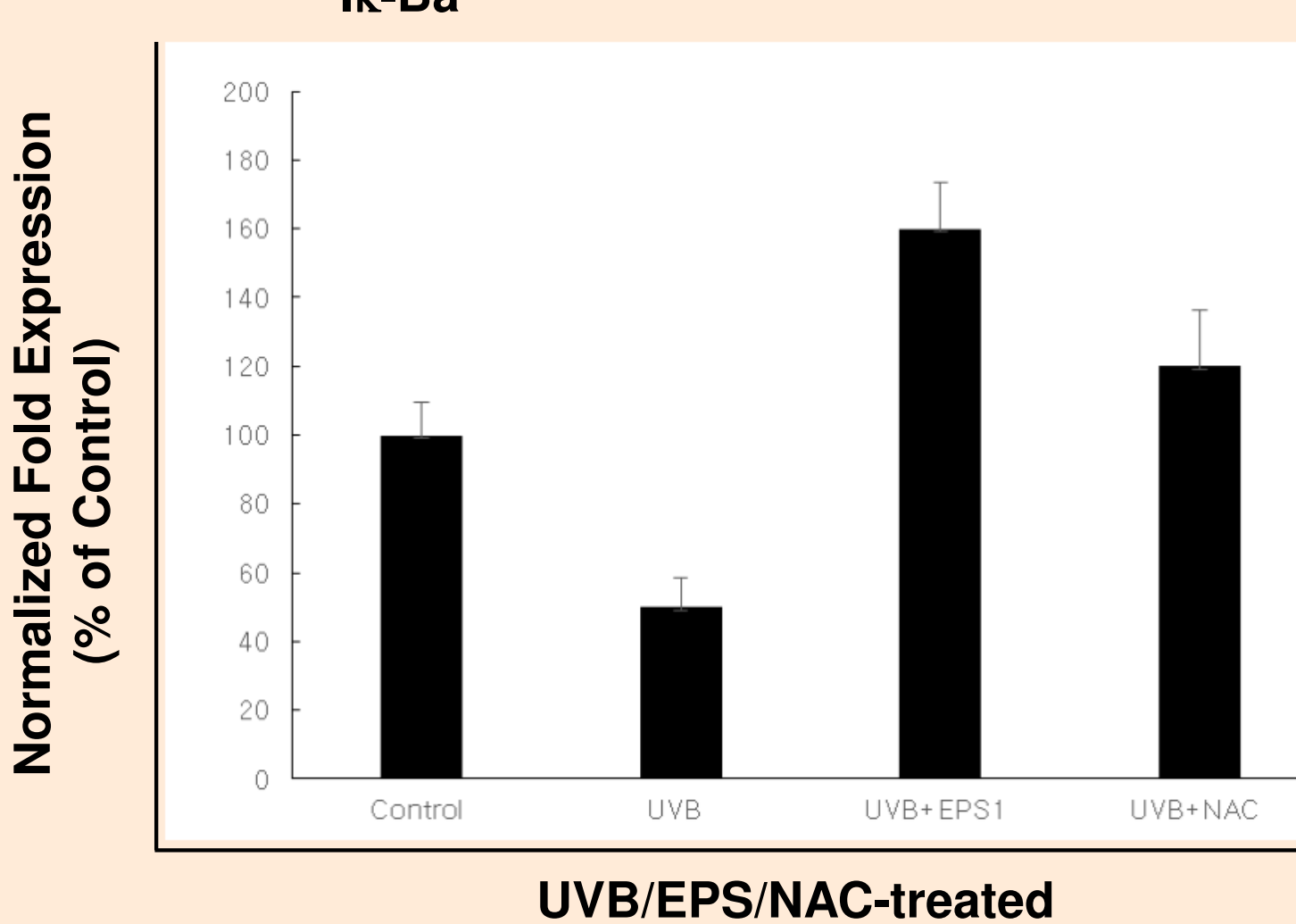

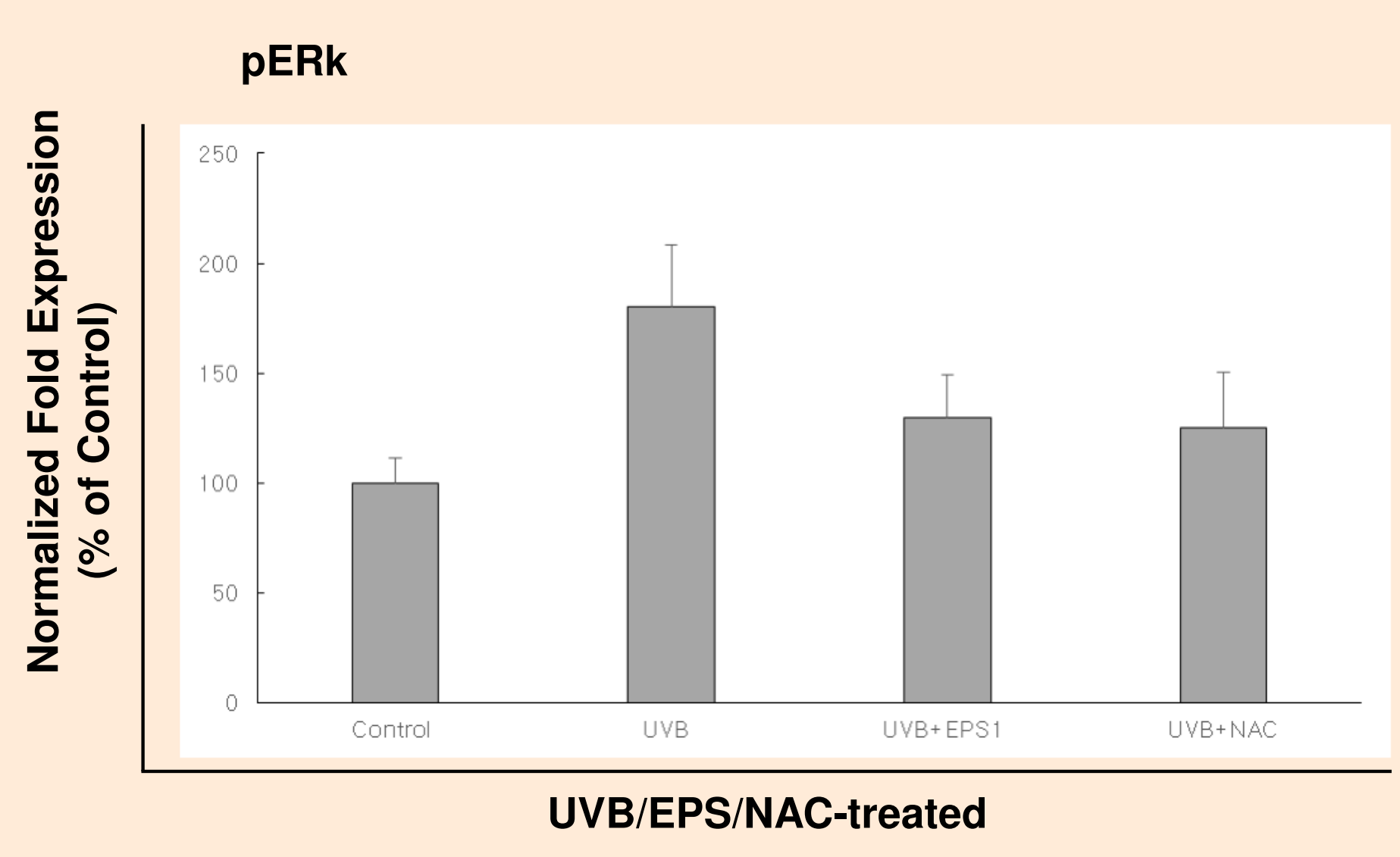

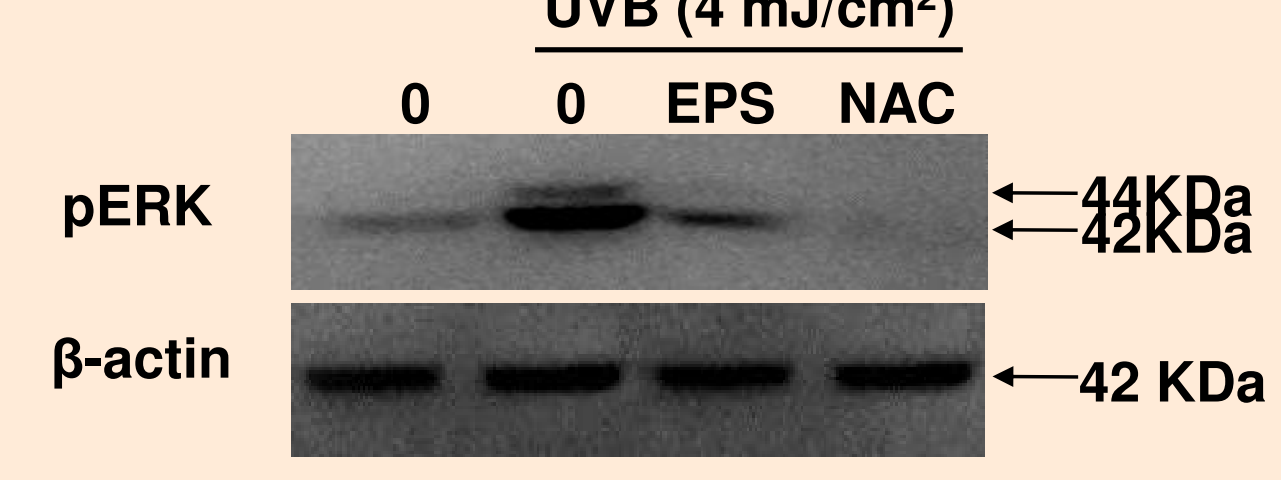

Figure 3. Modulation of inflammatory biomarkers by EPS in NHEKs

A. Expression levels of inflammatory biomarkers of IL-6, IL-8 and COX-2 were measured by RT-PCR experiments. B. Biomarkers for PERK cell signaling were checked with real-time PCR experiments. C. In Western blot analysis, UVB up-regulated PERK expression, which was reversed by EPS and NAC treatments. D. UVB-induced down-regulation of biomarkers for skin barrier (keratin 1, involucrin, filaggrin) were revsersed by
EPS and NAC treatments.

Figure 4. Modulation of pAKT and HIF-1a expression by EPS in NHEKs

In RT-PCR experiments, EPS and NAC showed activities to protect the UVB-induced upregulation of PAKT and HIF-1 $\alpha$ in NHEKs.

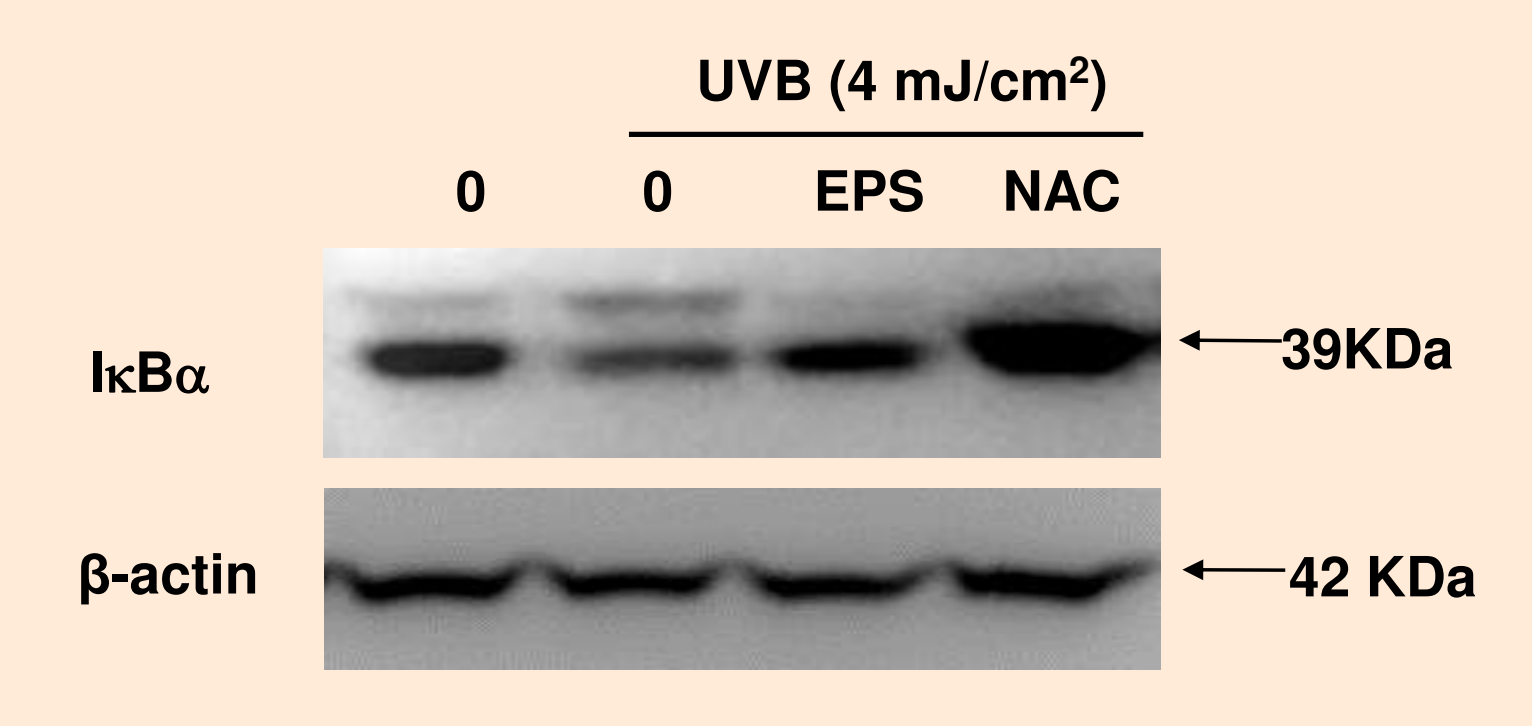

Figure 5. Modulation of IאB $\alpha$ expression, an inhibitor of NF-kB. by EPS in NHEKs

UVB-induced downregulation of $I_{K} B \alpha$ was reversed by EPS and NAC treatments in NHEKs. A. In real-time PCR, the

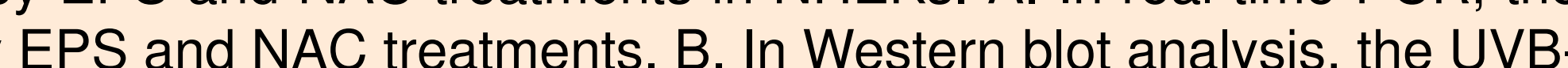
induced downregulation of IkB $\alpha$ was also reversed by EPS and NAC treatments.

\section{Summary}

- ROS play an important role in the pathogenesis of inflammation biomarker in keratinocytes via IL-6, IL-8 as well as MAPK and Akt-HIF-1 $\alpha$ pathways in keratinocytes.

- EPS is found to be a good candidate antioxidant to protect UVB-induced inflammation accompanying barrier dysfunction in keratinocytes. Further study is performing to develop a functional emollient including EPS as a new cosmeseutical product.

\section{Acknowledgement}

This project was supported by Basic Science Research Program through the National Research Foundation of Korea (NRF) funded by the Ministry of Education, Science and Technology (NRF-2015R1D1A1A01060023) 